\title{
Ischemic colitis caused by polycythemia vera: A case report and literature review
}

\author{
SHASHA ZHANG ${ }^{1}$, RUIXUE LAI $^{1}$, XUEQING GAO $^{1}$, YUFEI ZHAO $^{1}$, \\ YUE ZHAO $^{2}$, JIANHUA WU ${ }^{3}$ and ZHANJUN GUO ${ }^{1}$ \\ Departments of ${ }^{1}$ Rheumatology and Immunology, and ${ }^{2}$ Gastroenterology and Hepatology, \\ ${ }^{3}$ Animal Center, The Fourth Hospital of Hebei Medical University, Shijiazhuang, Hebei 050011, P.R. China
}

Received January 16, 2018; Accepted August 10, 2018

DOI: $10.3892 /$ etm.2018.6638

\begin{abstract}
Polycythemia vera (PV) is a chronic myeloproliferative disorder originating from hematopoietic stem cells and complicated by thrombosis and bleeding. This report describes a case of ischemic colitis (IC) caused by PV and includes a review of the relevant literature. The patient was a 59-year-old male with a history of PV who presented with abdominal pain and hematochezia. Colonoscopy and histopathological examination results indicated suspected ischemic bowel disease. Following experimental anticoagulant therapy for 7 days, the patient no longer experienced abdominal pain and hematochezia had resolved. Colonoscopy review showed no obvious anomalies 1 month later. These data demonstrated that PV is an uncommon cause of IC.
\end{abstract}

\section{Introduction}

Polycythemia vera (PV) is a rare and relatively poorly understood cause of chronic myeloproliferative disorder characterized by an increased red cell mass, circulating white blood cells (WBCs), and platelets; in this way, thrombosis and bleeding are the major complications associated with this disease. The most common sites of thrombosis and embolization are the intracranial artery, coronary artery, and abdominal blood vessels (1). The majority of patients with PV have Janus kinase 2 (JAK2) mutations, of which $~ 96 \%$ occur in JAK2V617F (exon 14) and $2 \%$ occur in JAK2 exon 12 (2). A diagnosis of PV is currently made in accordance with the 2016 World Health Organization criteria and is based on the composite assessment of clinical and laboratory features (http://www.bloodjournal.org/content/127/20/2391).

Correspondence to: Dr Zhanjun Guo, Department of Rheumatology and Immunology, The Fourth Hospital of Hebei Medical University, 12 Jiankang Road, Shijiazhuang, Hebei 050011, P.R. China

E-mail: zjguo5886@aliyun.com

Key words: polycythemia vera, ischemic colitis, abdominal pain, hematochezia, thrombus
Ischemic colitis (IC) can be described as an ischemic disease of the colon, which results from decreased blood flow due to various causes that leads to intestinal wall ischemia, injury or necrosis (3). The risk factors for IC mainly include diabetes, hypertension, dyslipidemia, peripheral vascular disease, aspirin administration, and digoxin or constipation-inducing medications (4). The main clinical manifestations include abdominal pain, diarrhea, and hematochezia (5). A reliable diagnosis of IC depends on the comprehensive evaluation of clinical symptoms, biochemical tests, radiological examinations, and endoscopic assessments (6). In certain cases, PV may cause mesenteric vascular thrombosis, which can lead to ischemic bowel disease, although uncommon. Cryer reported a case of pathologically confirmed ischemic bowel disease caused by PV in 1979 (7). In addition, Brada reported a case of IC caused by acute erythroid leukemia-induced PV in 1998 (8). In the present report, the third known case of PV-related ischemic bowel disease is described.

\section{Case report}

A 59-year-old Chinese male was admitted to The Fourth Hospital of Hebei Medical University (Shijiazhuang, China) on July 15, 2017 with complaints of abdominal pain for 2 weeks with 5 days of intermittent hematochezia. A review of the patient's medical history indicated a PV diagnosis by transient ischemic attack (TIA) accompanied by a significantly increased WBC count, hemoglobin (HGB) level, and platelet (PLT) count 9 years prior. Following treatment with regular aspirin enteric-coated tablets (100 mg/day) and hydroxyurea $(500 \mathrm{mg}$, three times per day), the patient's WBC count, HGB level and PLT count were within normal ranges. The cessation of aspirin and hydroxyurea administration between early February and early March 2017 induced increases in WBC count to $22.21 \times 10^{9} / 1$, red blood cell (RBC) count to $4.65 \times 10^{12} / 1$, HGB level to $165 \mathrm{~g} / 1$, and the PLT count to $467 \times 10^{9} / 1$. The reuse of aspirin enteric-coated tablets and hydroxyurea corrected the blood cell abnormalities; however, the patient complained of abdominal pain characterized by sustained spasmodic pain in the middle abdomen, which worsened following eating, 10 days prior to local hospitalization. These symptoms were accompanied by small amounts of bloody stools with mucous 7-8 times per day, 1 day prior to local hospitalization. Routine blood 

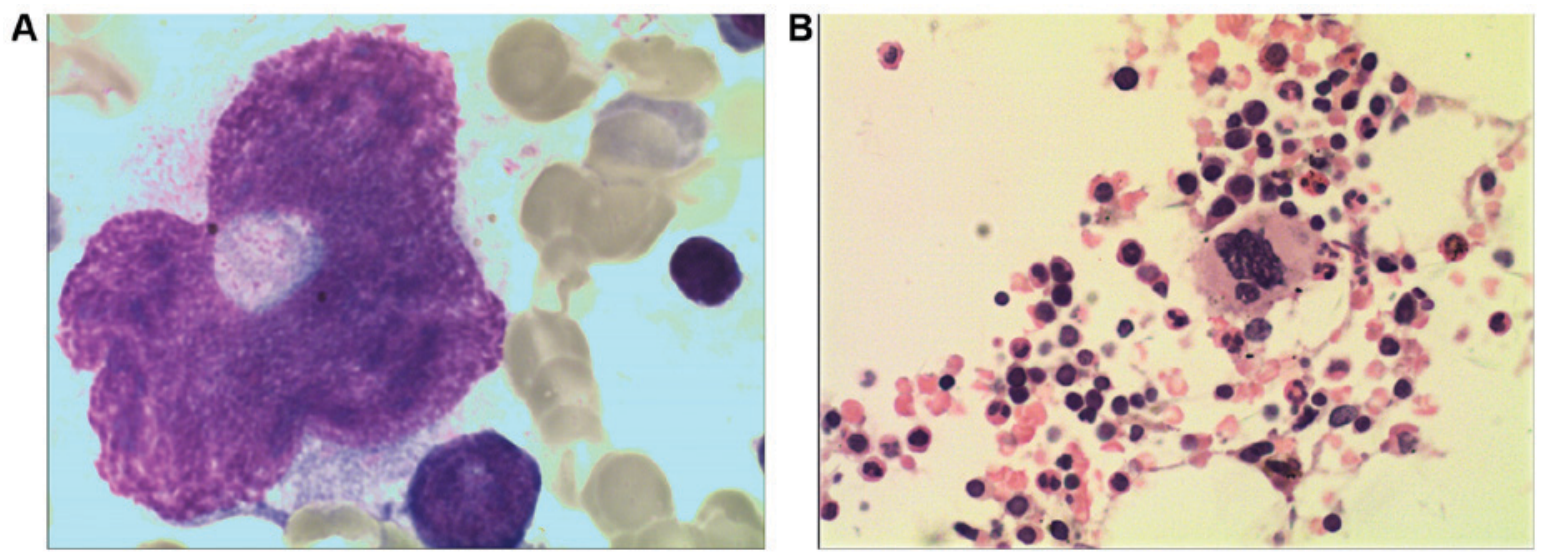

Figure 1. Pathological changes. (A) Bone marrow cytology showed an increased red ratio. Bone marrow extracted from the anterior superior iliac spine was smeared, aired, and stained using the Wright-Giemsa method (magnification, x1,000). (B) Bone marrow biopsy was indicative of myeloproliferative disease with pathological changes. Bone marrow was paraffin embedded following fixing with $10 \%$ formalin, dehydrated, and sectioned (2-4- $\mu \mathrm{m})$ for hematoxylin and eosin staining (magnification, $\mathrm{x} 400$ ).

tests revealed that the patient's WBC count was $15.94 \times 10^{9} / 1$, the RBC count was $4.27 \times 10^{12} / 1$, the HGB level was $151 \mathrm{~g} / 1$, the PLT count was $403 \times 10^{9} / 1$, and the D-dimer level was $1 \mathrm{mg} / 1$. The subsequent treatment of hemostasis and antibiotics did not improve the patient's symptoms of intermittent abdominal pain or hematochezia following colonoscopy revealing colonic lesions; therefore, the patient was transferred to The Fourth Hospital of Hebei Medical University for further treatment with the following clinical characteristics.

On physical examination, the patient's blood pressure was $110 / 69 \mathrm{mmHg}$, pulse was 85 beats/min, respiration rate was $21 \mathrm{times} / \mathrm{min}$, and body temperature was $36.9^{\circ} \mathrm{C}$. The abdomen was soft and there was no tenderness with active bowel sounds.

The laboratory examination revealed a WBC count of $10.55 \times 10^{9} / 1$, an RBC count of $3.35 \times 10^{12} / 1$, an HGB level of $115.7 \mathrm{~g} / 1$, a PLT count of $442 \times 10^{\%} / 1$, and a D-dimer level of $0.156 \mathrm{mg} / \mathrm{l}$. Tumor markers, including carcinoembryonic antigen (CEA), carbohydrate antigen 19-9 (CA19-9), carbohydrate antigen 72-4 (CA72-4), and hepatorenal function, were all within the normal ranges. The fecal occult blood test was positive, and the bone marrow cytology and biopsy showed an increased red ratio. The pathological changes were indicative of myeloproliferative disease, and the patient was JAK2V617F-positive (Fig. 1A and B).

An enhanced abdominal computed tomography (CT) scan revealed a thickened transverse colon wall, suspected to be due to malignant lesions (Fig. 2A and B). The colonoscopy examination revealed segmental and superficial ulcers, mucosal hyperemia in the cecum, and hepatic flexure at the transverse colon, which were involved either all around or on one side of the intestinal wall. The boundary between the lesion and the normal intestinal mucosa was clear and the remaining colon mucosa was smooth (Fig. 3A-C). The colonoscopy yielded a diagnosis of colonic lesions, either due to Crohn's disease (CD) or IC, and the histopathological diagnosis indicated mucosal chronic inflammation and inflammatory granulation.

The patient was treated with mesalazine enteric-coated tablets ( $1 \mathrm{~g}$ ) four times per day for 2 days without symptomatic relief. The subsequent subcutaneous injection of low-molecular-weight heparin (100 IU/kg) once every $12 \mathrm{~h}$ was performed for suspected IC. The patient's abdominal pain and hematochezia were relieved 1 day later. In addition, the fecal occult blood test was negative 2 days later. Superior and inferior mesenteric artery CT angiography was performed, which indicated arteriosclerosis in the superior and inferior mesenteric artery (Fig. 4). It was recommended that the patient undergo mesenteric angiography, however, the patient refused the procedure for financial reasons. The patient was discharged from hospital 7 days following anticoagulant therapy with continued administration of aspirin enteric-coated tablets (100 $\mathrm{mg} /$ day) and hydroxyurea (500 mg, three times per day), and with a regular review of routine blood tests. A colonoscopy review was performed 1 month later and the entire colon mucous membrane had returned to normal (Fig. 5A-C).

\section{Discussion}

The enhanced abdominal CT scan performed in hospital detected possible malignant lesions of the transverse colon, however, the patient complained only of abdominal pain and hematochezia with no alarming symptoms of weight loss, weakness, or anorexia; the patient's intestinal tumor markers were normal. In addition, the histopathology results did not support a diagnosis of colon cancer. Therefore, the patient was treated with mesalazine enteric-coated tablets first, based on the results of the colonoscopy examination, which were indicative of either CD or IC.

$\mathrm{CD}$ is an inflammatory immune-related disease, which usually manifests between 15-30 years of age, and its clinical features largely depend on disease location (9). The typical presentations include abdominal pain, diarrhea, weight loss, and an abdominal mass, and the complications include strictures, abscesses, and fistulas (10). The stools are predominantly loose without blood, with the exception that lesions are involved in the lower colon and rectum (11). Endoscopic features include discontinuous ulcerations (skip lesions), a cobblestone pattern, deep ulcerations, and strictures (9). The results of histopathology typically reveal the presence of a non-caseating granuloma. It was difficult to distinguish 

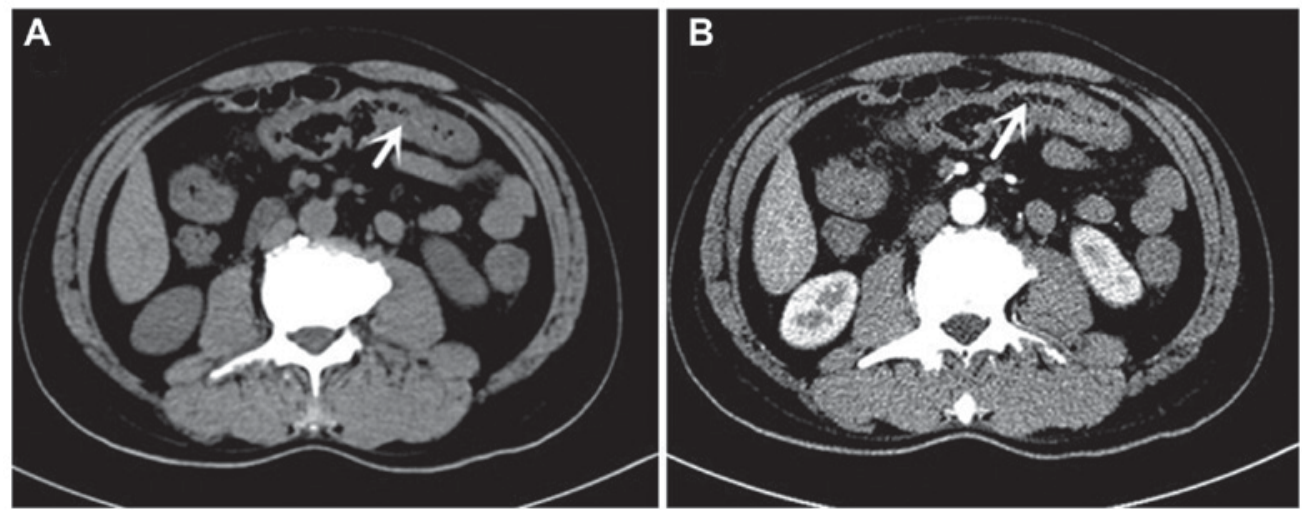

Figure 2. Abdominal CT scan prior to treatment. (A) Plain CT scan revealed a thickened transverse colon wall; (B) enhanced CT scan revealed irregular enhancement in the transverse colon wall (white arrows). CT, computed tomography.

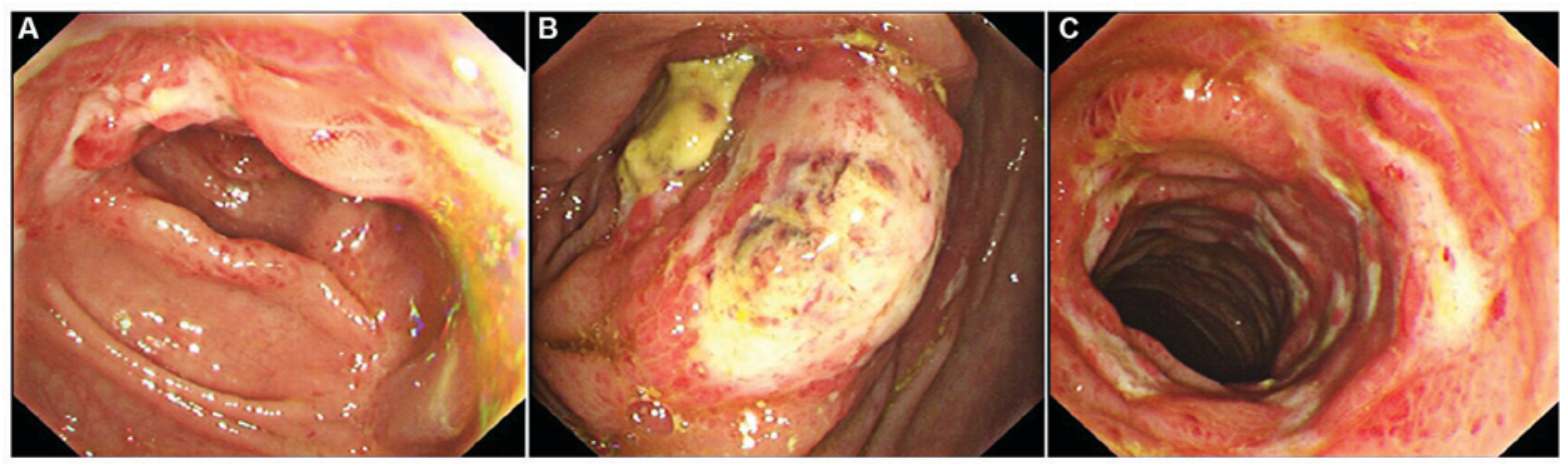

Figure 3. Manifestations of colonoscopy examination prior to treatment. Segmental and superficial ulcers, mucosal hyperemia were observed in the cecum, with hepatic flexure at the transverse colon. (A) Cecum. (B) Ulcers involved on one side of the hepatic flexure. (C) Ulcers involved all around the transverse colon.

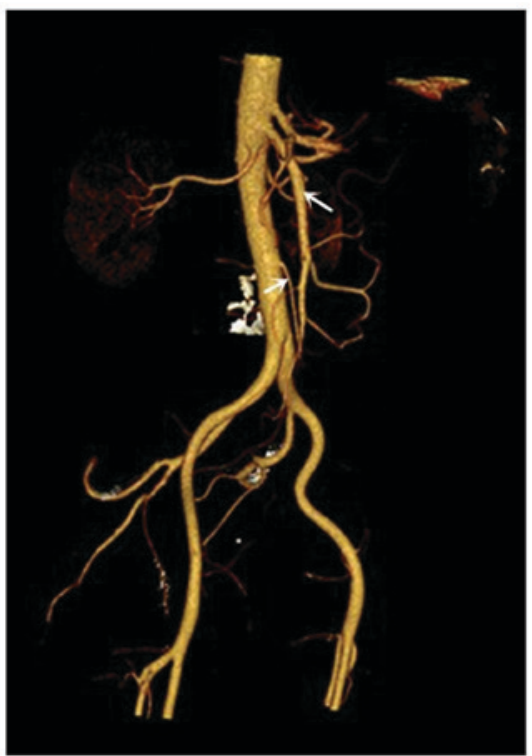

Figure 4. Superior and inferior mesenteric artery computed tomography angiograph in the process of treatment. Arteriosclerosis was present in the superior and inferior mesenteric artery. The white arrows point to the superior and inferior mesenteric artery, respectively.

between $\mathrm{CD}$ and IC from the colonoscopy and histopathology findings of the patient examined in the present report.
Although IC mainly occurs in the left hemi-colon (12), Cryer reported a case of pathologically confirmed ischemic bowel disease caused by PV, where the patient's lesion was located in the right hemi-colon (7); this is similar to the case reported here. Therefore, experimental therapy of low-molecular-weight heparin was performed following treatment with mesalazine enteric-coated tablets, based on the risk factors of ischemic bowel disease including a history of PV, the cessation of aspirin enteric-coated tablets and hydroxyurea prior to onset of illness, and the increased WBC count and PLT levels when hematochezia was identified. In addition, the patient had a TIA 9 years previously and the incidence of vascular events is approximately doubled in patients who have a history of previous thrombosis (13). Furthermore, the following clinical features supported IC: Disease had a sudden onsongside persistent spasmodic abdominal pain followed by hematochezia; the patient's abdominal pain was worse on eating and did not ease on defecation; colonoscopy examination revealed segmental and superficial ulcers, mucosal hyperemia in the cecum, and hepatic flexure at the transverse colon, which were either involved all around or on one side of the intestinal wall; the boundary between the lesion and the normal intestinal mucosa was clear.

When D-dimer levels are $>0.9 \mathrm{mg} / \mathrm{l}$, the specificity and sensitivity of a diagnosis of ischemic bowel disease are 82 and $60 \%$, respectively (14). The patient had high D-dimer levels of 


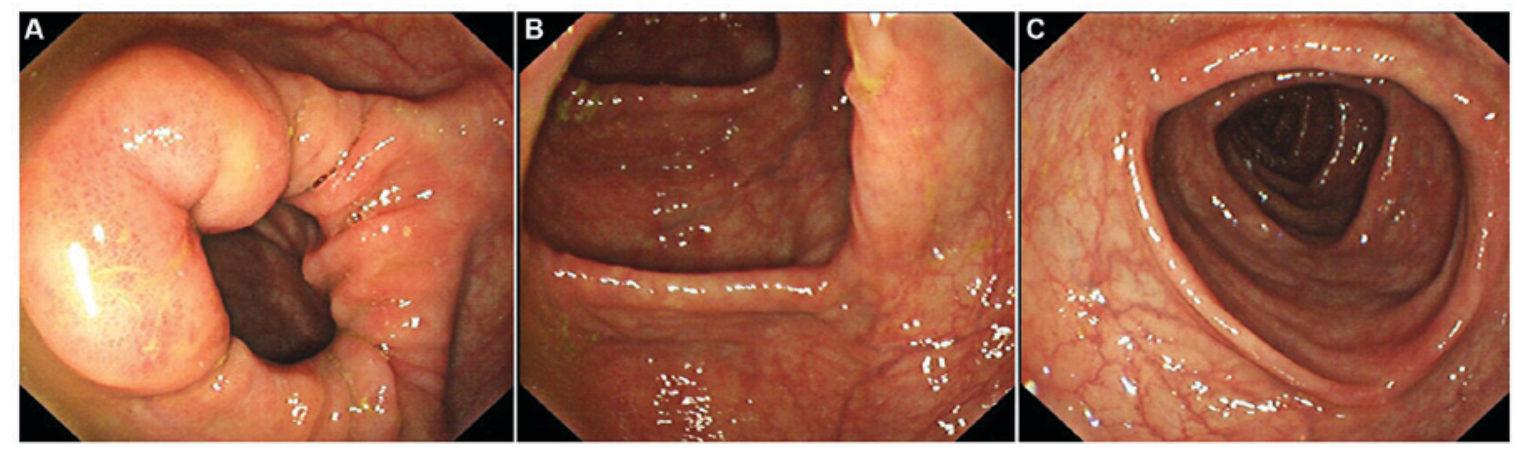

Figure 5. Colonoscopy review 1 month following discharge. The entire colon mucous membrane had returned to normal. (A) Cecum. (B) Hepatic flexure. (C) Transverse colon.

$1 \mathrm{mg} / \mathrm{l}$ when he had bloody stools, which also supports a diagnosis of ischemic bowel disease. Although CT angiography has $94 \%$ specificity and $96 \%$ sensitivity to the diagnosis of occlusive mesenteric ischemia for the large mesenteric artery, the observation of branches below the third level of the mesenteric artery trunk with CT angiography is not reliable (15). Selective mesenteric angiography is the gold standard for a diagnosis of ischemic bowel disease with a sensitivity of 90-100\% even for the small mesenteric artery (16), however, the patient refused mesenteric angiography for financial reasons following a negative CT scan for thrombus. Following experimental anticoagulation therapy, the patient no longer reported suffering from abdominal pain or hematochezia, and the fecal occult blood test was negative. The patient continued receiving aspirin enteric-coated tablets (100 $\mathrm{mg} /$ day) and hydroxyurea (500 mg, three times per day), and the patient underwent regular review of routine blood tests following discharge from hospital. The colonoscopy review showed no obvious anomalies 1 month later, which enabled the exclusion of nonsteroidal anti-inflammatory drug-related enteropathy caused by aspirin.

This is the third reported case of IC caused by PV. The previous two cases were characterized by increased leukocyte and hematocrit counts, whereas the case described here was characterized by elevated leukocyte counts and platelet levels. It is reported that a JAK2 V617 mutation affects the number and activation of WBCs (17). Landolfi reported that the risk of thrombosis was significantly increased when the WBC count was $>10 \times 10^{9} / 1$ in patients with PV (13), although the underlying mechanism of thrombosis remains to be fully elucidated (18).

In conclusion, the present report describes a case of a patient with IC caused by PV with elevated leukocyte counts and platelet levels.

\section{Acknowledgements}

The authors would like to thank Professor Liu Yueping (Department of Pathology) and Professor Wang Qi (Department of Radiology) in The Fourth Hospital of Hebei Medical University for their kind assistance with the pathological and radiographic images.

\section{Funding}

The present study was supported by the Science and Technology Project of Hebei (grant no. 162777114D).

\section{Availability of data and materials}

The datasets used and/or analyzed during the current study are available from the corresponding author on reasonable request.

\section{Authors' contributions}

SZ collected the data and wrote the manuscript. RL collected the pathological data. XG collected the radiographic data. YufZ contributed to the study design and manuscript review. YueZ collected the endoscopic data. JW collected and revised the figures. ZG contributed to project design, data collection and manuscript writing. All authors read and approved the final manuscript. The authors agreed to be accountable for all aspects of the study, ensuring that questions related to the accuracy or integrity of any part of the study are appropriately investigated and resolved.

\section{Ethics approval and consent to participate}

All procedures were supervised and approved by the Ethics Committee of The Fourth Hospital of Hebei Medical University. The patient provided written informed consent prior to enrolment in the study.

\section{Patient consent for publication}

Not applicable.

\section{Competing interests}

The authors declare that they have no competing interests.

\section{References}

1. Finazzi G and Barbui T: Evidence and expertise in the management of polycythemia vera and essential thrombocythemia. Leukemia 22: 1494-1502, 2008.

2. Tefferi A and Barbui T: Polycythemia vera and essential thrombocythemia: 2017 update on diagnosis, risk-stratification, and management. Am J Hematol 92: 95-108, 2017.

3. Chen M, Remer EM, Liu X, Lopez R and Shen B: Identification of the distinguishing features of Crohn's disease and ischemic colitis using computed tomographic enterography. Gastroenterol Rep (Oxf) 5: 219-225, 2017.

4. FitzGerald JF and Hernandez Iii LO: Ischemic colitis. Clin Colon Rectal Surg 28: 93-98, 2015. 
5. Bradbury MS, Kavanagh PV, Bechtold RE, Chen MY, Ott DF, Regan FD and Weber TM: Mesenteric venous thrombosis: Diagnosis and noninvasive imaging. Radiographics 22: 527-541, 2002.

6. Stamatakos M, Douzinas E, Stefanaki C, Petropoulou C, Arampatzi H,Safioleas C, Giannopoulos G, Chatziconstantinou C, Xiromeritis C and Safioleas M: Ischemic colitis: Surging waves of update. Tohoku J Exp Med 218: 83-92, 2009.

7. Polycythemia and abdominal pain. Am J Med 66: 321-330, 1979.

8. Brada SJ, de Wolf JT, Poppema S and Vellenga E: Ischaemic colitis and lung infiltrates caused by extramedullary haematopoiesis in a patient with an acute erythroid leukaemia following polycythaemia vera. Neth J Med 52: 142-146, 1998.

9. Laass MW, Roggenbuck D and Conrad K: Diagnosis and classification of Crohn's disease. Autoimmun Rev 13: 467-471, 2014.

10. Baumgart DC and Sandborn WJ: Inflammatory bowel disease: Clinical aspects and established and evolving therapies. Lancet 369: 1641-1657, 2007.

11. Mazal J: Crohn disease: Pathophysiology, diagnosis, and treatment. Radiol Technol 85: 297-320, 2014.

12. Flynn AD and Valentine JF: Update on the diagnosis and management of colon ischemia. Curr Treat Options Gastroenterol 14: 128-139, 2016.

13. Landolfi R, Di Gennaro L, Barbui T, De Stefano V, Finazzi G, Marfisi R, Tognoni G and Marchioli R; European Collaboration on Low-Dose Aspirin in Polycythemia Vera (ECLAP): Leukocytosis as a major thrombotic risk factor in patients with polycythemia vera. Blood 109: 2446-2452, 2007.
14. Block T, Nilsson TK, Björck M and Acosta S: Diagnostic accuracy of plasma biomarkers for intestinal ischaemia. Scand J Clin Lab Invest 68: 242-248, 2008.

15. Kirkpatrick ID, Kroeker MA and Greenberg HM: Biphasic CT with mesenteric CT angiography in the evaluation of acute mesenteric ischemia: Initial experience. Radiology 229: 91-98, 2003.

16. Brandt LJ and Boley SJ: AGA technical review on intestinal ischemia. American Gastrointestinal Association. Gastroenterology 118: 954-968, 2000.

17. De Stefano V, Za T, Rossi E, Vannucchi AM, Ruggeri M, Elli E, Micò C, Tieghi A, Cacciola RR, Santoro C, : Leukocytosis is a risk factor for recurrent arterial thrombosis in young patients with polycythemia vera and essential thrombocythemia. Am J Hematol 85: 97-100, 2010.

18. Falanga A, Marchetti M, Vignoli A, Balducci D and Barbui T: Leukocyte-platelet interaction in patients with essential thrombocythemia and polycythemia vera. Exp Hematol 33: 523-530, 2005. 